\title{
Geomagnetic Secular Variation and Poloidal Magnetic Fields Produced by Convectional Motions in the Earth's Core
}

\author{
By Takesi Nagata \\ Geophysical Institute, University of Tokyo \\ and \\ Tsuneji RIKITAKE \\ Earthquake Research Institute, University of Tokyo \\ (Read November 22, 1961 ; Received December 1, 1961)
}

\begin{abstract}
A theory of electromagnetic induction by a convectional fluid motion within the earth's core is attempted in the hope of accounting for the localized intense secular variation in the geomagnetic field as has been found in the Antarctic area. If a toroidal magnetic field of 300 gauss at maximum is supposed to exist in the core, a steady convectional motion described by a spherical harmonic of order 5 and degree 5 can give rise to a poloidal magnetic field (degree 6, order 5 ) of which the Gaussian coefficient of the magnetic potential amounts to $0.045 \Gamma$ at the earth's surface provided the radial velocity of the motion is taken as $0.01 \mathrm{~cm} / \mathrm{sec}$.

A study of the growth of the field tells us that a secular change of the order of $100 \mathrm{r} / \mathrm{yr}$ is expected as long as a velocity of $0.1 \mathrm{~cm} / \mathrm{sec}$ is assumed. This order of velocity would not be impossible for such a localized motion as considered here.
\end{abstract}

\section{Introduction}

Since Elsasser ${ }^{(1)}$ emphasized the importance of electromagnetic induction within moving material in the earth's core, the possibility of accounting for the non-dipole part of the geomagnetic field and its secular variation through the induction process has drawn attention of a number of authors. For example, Bullard ${ }^{(2)}$ has considered induction in eddies idealized as rotating spheres in order to account for the secular variation observed in South Africa and its neighbourhood. In his result has been suggested an important conclusion that a toroidal field much stronger than the dipole one is likely to be in existence in the core.

Now since IGY, a fairly good network of observations of geomagnetic secular variation has been extended over the whole surface of the earth, and various features of geomagnetic secular variation, especially in the southern hemisphere where only very little had been known until that time, have steadily been clarified. As pointed out and confirmed by one of the present writers $^{(3),(4),(5)}$, a remarkable fact obtained in the recent studies on the secular variation is that an anomalously large secular variation 
amounting to about $200 \mathrm{r} /$ year in rate of change is taking place in the East Antarctic area.

It has been concluded that this large secular variation has continued at least during the past 20 years. The isoporic chart of $\dot{Z}$ for the period of 1955-60 in the southern hemisphere is reproduced in Fig. 1, where the magnitudes of $\dot{X}$ and $\dot{Y}$ are also illustrated by vector arrows.

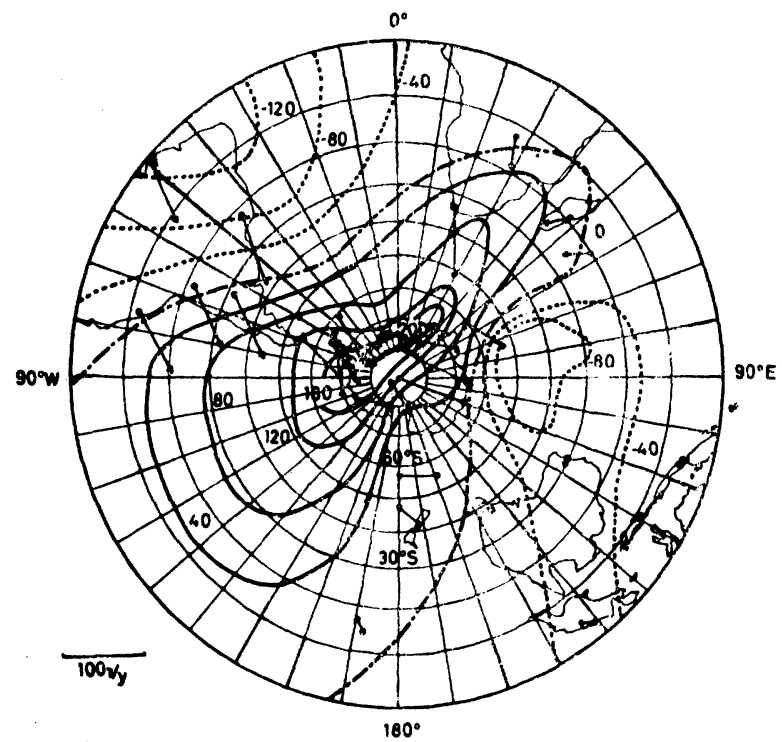

Fig. 1. Isoporic chart of $\dot{Z}$ for $1955-60$ in unit of $\gamma /$ year in the southern hemisphere. Full circles show observing points and arrows represent $\dot{H}=(\dot{X}, \dot{Y})$ at those points.

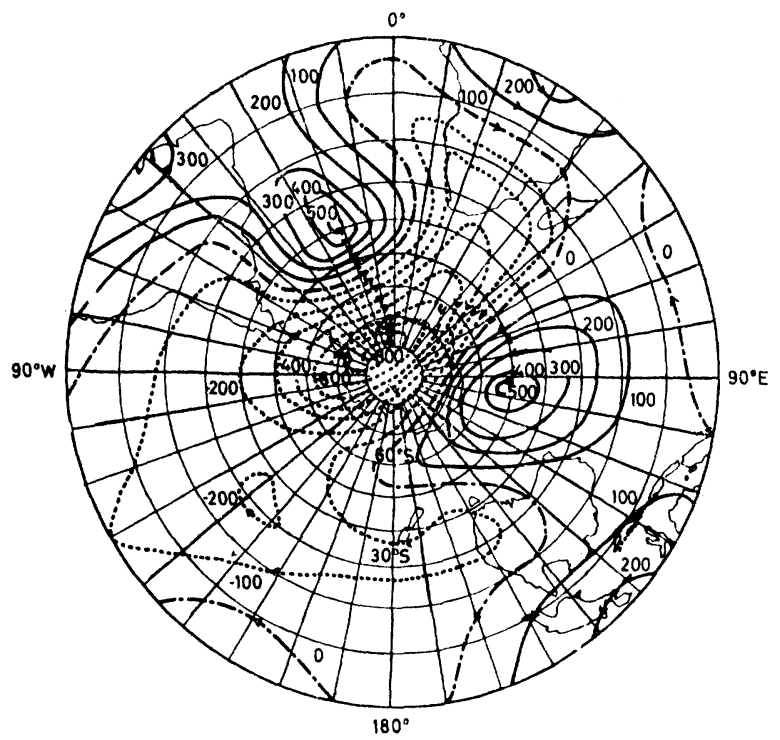

Fig. 2. Equivalent current system of the geomagnetic secular variation at the depth of $2900 \mathrm{~km}$, in unit of $10^{4}$ Ampere/year. 
The isoporic chart being subject to a spherical harmonic analysis of 6 in its highest degree, the equivalent current system of the variation assumed at the depth of $2900 \mathrm{~km}$ (namely, on the surface of the earth's core) is obtained as illustrated in Fig. 2. As shown in this figure and as confirmed by numerical values of the spherical harmonic coefficients, the localized distribution of the intense secular variation in the Antarctic area can roughly be represented by harmonics of 4,5 and 6 in their degree.

Then, the question of how such a localized intense secular variation can theoretically be explained may rise again. As a cause of secular variation, the effect of the westward drift of non-dipole components of the geomagnetic field has widely been accepted $^{(6),(7)}$. Provided that the origin and a sufficiently long life of non-dipole magnetic fields produced in the earth's core are reasonably explained, a possibility of the westward drift of these fields can be theoretically understood ${ }^{(7)}$. According to the result of analysis, a tendency of westward drift of 0.18 degree/year in average velocity (ranging from 0 to 0.6 degree/year) can be detected for harmonics of $n=2 \sim 4$, but not for harmonics higher than 4 in their degree. Further, comparing the localized secular variation directly to the non-dipole field ${ }^{(8)}$ in the Antarctic area, it is found that the hypothesis of westward drift of the non-dipole field can not account for the cause of the secular variation.

Then, the most probable theoretical explanation of this phenomenon may be to assume upwelling of a part of strong toroidal magnetic field caused by a particular fluid motion within the earth's core, as already suggested ${ }^{(4)}$.

Bullard's work ${ }^{(2)}$ for interpreting the South African secular variation was along this direction of research. The induction process by rigid rotators embedded in stationary conductors, such as assumed in the Bullard's first paper, has been later examined extensively by Herzenberg and Lowes ${ }^{(9)}$. In contrast to rigid rotator models, more realistic models based on interaction between magnetic field and fluid motion have been also studied. Coulomb $b^{(10)}$ has discussed the advection of the poloidal field on the basis of a simple model for upwelling motion in the core. The idea that a toroidal field which is presumably strong at some depth in the core can be brought up by convection has been discussed by Parker ${ }^{(11)}$. Although such a distorsion of a toroidal field by convectional motion seems to have an important bearing on the dynamo process which maintains the main geomagnetic field, no exact theory with detailed estimate has been so far available except the work made by Allan and Bullard ${ }^{(12)}$, of which only a short note has been published. It is the intention of this paper to see what the extent of the poloidal field will be outside the core when a fluid motion of convection type and a toroidal field are specified in the core. And it is hoped to find some clue for explaining the intense variation in the Antarctic area.

\section{Theory}

The induction equation for magnetic field $\vec{h}$ is given by

$$
\partial \vec{h} / \partial t-(4 \pi \sigma)^{-1} \nabla^{2} \vec{h}=\operatorname{curl}\left(\vec{v} \wedge \vec{H}_{0}\right)
$$


where $\sigma, \vec{v}$ and $\vec{H}_{0}$ denote the electrical conductivity, velocity of the fiuid motion and stationary magnetic field. It is not intended here to take the equation of motion into account, so that the present treatment is not really hydromagnetic. Some discussion of the hydromagnetic effect will be made later.

The fluid motion is assumed as a poloidal one. The $r, \theta$ and $\phi$ components of the velocity are given as

$$
\vec{v}=\left\{\begin{array}{l}
-n(n+1) \xi_{n}^{m}(r) r^{n-1} Y_{n}^{m} \\
-\left[r \frac{d \xi_{n}^{m}}{d r}+(n+1) \xi_{n}^{m}\right] r^{n-1} \frac{\partial Y_{n}^{m}}{\partial \theta} \\
-\left[r \frac{d \xi_{n}^{m}}{d r}+(n+1) \xi_{n}^{m}\right] r^{n-1} \frac{\partial Y_{n}^{m}}{\sin \theta \partial \phi}
\end{array}\right.
$$

where $Y_{n}^{m}$ stands for either $P_{n}^{m}(\cos \theta) \cos m \phi$ or $P_{n}^{m}(\cos \theta) \sin m \phi$ and the origin of the polar coordinate is taken at the centre of the core.

The theory of the origin of the earth's magnetic field suggests that there is a very strong toroidal field, presumably as strong as several hundred gauss, in the core. Let us then assume that the stationary field is given as

$$
\vec{H}_{0}=\left\{\begin{array}{l}
0 \\
0 \\
T_{2}(r) r^{2} \frac{d P_{2}}{d \theta}
\end{array}\right.
$$

The induction process due to $\vec{v}$ and $\vec{H}_{0}$, that is shown by the righthand-side of equation (1), then gives rise to magnetic fields of various types.

They are

$$
\begin{array}{llll}
\text { Poloidal : } & S_{n-1}^{m}, & S_{n+1}^{m} & \\
\text { Toroidal : } & T_{n-2}^{m}, & T_{n}^{m}, & T_{n+2}^{m}
\end{array}
$$

where $n$ and $m$ denote respectively the degree and order of spherical surface harmonics involved. The fields of toroidal type do not appear outside the core provided the conductivity in the mantle is assumed to be very poor, so that our concern is confined to the poloidal ones which are readily written as

$S_{n-1}^{m}$ field :

$$
\vec{h}_{n-1}^{m}=\left\{\begin{array}{l}
-(n-1) n s_{n-1}^{m}(r) r^{n-2} \widetilde{Y}_{n-1}^{m} \\
-\left[r \frac{d s_{n-1}^{m}}{d r}+n s_{n-1}^{m}\right] r^{n-2} \frac{\partial \widetilde{Y}_{n-1}^{m}}{\partial \theta} \\
-\left[r \frac{d s_{n-1}^{m}}{d r}+n s_{n-1}^{m}\right] r^{n-2} \frac{\partial \widetilde{Y}_{n-1}^{m}}{\sin \theta \partial \phi}
\end{array}\right.
$$

$S_{n+1}^{m}$ field:

$$
\vec{h}_{n+1}^{\prime \prime \prime}=\left\{\begin{array}{l}
-(n+1)(n+2) s_{n+1}^{m}(r) r^{n} \widetilde{Y}_{n+1}^{m} \\
-\left[r \frac{d s_{n+1}^{m}}{d r}+(n+2) s_{n+1}^{m}\right] r^{n} \frac{\partial \widetilde{Y}_{n+1}^{m}}{\partial \theta} \\
-\left[r \frac{d s_{n+1}^{m}}{d r}+(n+2) s_{n+1}^{m}\right] r^{n} \frac{\partial \widetilde{Y}_{n+1}^{m}}{\sin \theta \partial \phi}
\end{array}\right.
$$


where

$$
\widetilde{Y}_{n}^{m}=\partial Y_{n}^{m} / \partial \phi
$$

Introducing (4) and (5) to (1) of which the righthand-side has been calculated from (2) and (3), we obtain differential equations for radial parts $s_{n-1}^{m}$ and $s_{n+1}^{m}$ as

$$
\begin{aligned}
& \frac{d^{2} s_{n-1}^{m}}{d r^{2}}+\frac{2 n}{r} \frac{d s_{n-1}^{m}}{d r}-k^{2} s_{n-1}^{m}=f_{n-1}(r) \\
& \frac{d^{2} s_{n+1}^{m}}{d r^{2}}+\frac{2(n+2) d s_{n+1}^{m}}{d r}-k^{2} s_{n+1}^{m}=f_{n+1}(r)
\end{aligned}
$$

where

$$
k^{2}=4 \pi \sigma p
$$

and $p$ is algebraic operator $\partial / \partial t . f_{n-1}$ and $f_{n+1}$ are derived from the induction effect and given as

$$
\begin{aligned}
& f_{n-1}(r)=-3 \frac{n+1}{n-1} \frac{n+m}{2 n+1} 4 \pi \sigma \xi_{n}^{m}(r) T_{2}(r) r^{2} \\
& f_{n+1}(r)=-3 \frac{n}{n+2} \frac{n-m+1}{2 n+1} 4 \pi \sigma \xi_{n}^{m}(r) T_{2}(r)
\end{aligned}
$$

The solution of differential equation (7), that is finite at $r=0$, is obtained as

$$
\begin{array}{r}
s_{n-1}^{m}(r)=r^{-n+1 / 2}\left[A_{n-1} I_{n-1 / 2}(k r)+I_{n-1 / 2}(k r) \int r^{n+1 / 2} K_{n-1 / 2}(k r) f_{n-1}(r) d r\right. \\
\left.-K_{n-1 / 2}(k r) \int r^{n+1 / 2} I_{n-1 / 2}(k r) f_{n-1}(r) d r\right]
\end{array}
$$

in which $A_{n-1}$ is a free constant which is to be determined by the boundary conditions. $I$ and $K$ are modified Bessel functions.

If we ignore the electrical conductivity in the mantle, the potential of the magnetic field corresponding to (4) is given as

$$
w_{n-1}^{m}=a(r / a)^{-n} i_{n-1}^{m} \widetilde{Y}_{n-1}^{m}
$$

outside the core, where $a$ denotes the core's radius. $i_{n-1}^{m}$ is then the coefficient for the magnetic potential originating from the interior of the core. The magnetic field is then obtained as

$$
\vec{h}_{n-1}^{m}= \begin{cases}n\left(\frac{r}{a}\right)^{-n-1} & i_{n-1}^{m} \widetilde{Y}_{n-1}^{m} \\ -\left(\frac{r}{a}\right)^{-n-1} & i_{n-1}^{m} \frac{\partial \widetilde{Y}_{n}^{m}}{\partial \theta} \\ -\left(\frac{r}{a}\right)^{-n-1} & i_{n-1}^{m} \frac{\partial \widetilde{Y}_{n-1}^{m}}{\sin \theta \partial \phi}\end{cases}
$$

At $r=a$, the continuity condition for the normal and tangential components of the magnetic field (the magnetic permeability is assumed as unity in electromagnetic unit) then leads to 


$$
\begin{aligned}
& -(n-1) n s_{n-1}^{m}(a) a^{n-2}=n i_{n-1}^{m} \\
& \left(r \frac{d s_{n-1}^{m}}{d r}+n s_{n-1}^{m}\right)_{r=a} a^{n-2}=i_{n-1}^{m}
\end{aligned}
$$

from which, eliminating $i_{n-1}^{m}$, we obtain

$$
\left[r \frac{d s_{n-1}^{m}}{d r}+(2 n-1) s_{n-1}^{m}\right]_{r=a}=0
$$

This is the condition by which constant $A_{n-1}$ is to be determined. Before we proceed to determine $A_{n-1}$, however, we have to perform the integrations involved in (12). Since no detailed distributions of $\xi_{n}^{m}(r)$ and $T_{2}(r)$ have been so far known, it is necessary to make some assumption concerning these distributions. According to Bullard and Gellman ${ }^{(13)}$, the toroidal field in question is very small at the core-mantle boundary getting larger with the increase in depth. In one of their examples, the maximum of the field intensity occurs somewhere at the depth of $0.4 a$. Because of the solid inner core, however, it would seem more likely to suppose that the maximum occurs at a shallower depth.

For the purpose of making the integrals in (12) tractable, it is assumed here that the toroidal field is zero except in a spherical shell between $r=c$ and $r=c+\Delta c, \Delta c$ being small compared to $c . \quad c=0.8 a$ and $\Delta c=0.1 a$ as chosen in the later numerical work would be one of the likely combinations for actual values. In that case, (12) reduces to

$$
\begin{array}{r}
s_{n-1}^{m}(r)=r^{-n+1 / 2}\left[A_{n-1} I_{n-1 / 2}(k r)+c^{n+1 / 2} \Delta c \bar{f}_{n-1}\left\{I_{n-1 / 2}(k r) K_{n-1 / 2}(k c)\right.\right. \\
\left.\left.-K_{n-1 / 2}(k r) I_{n-1 / 2}(k c)\right\}\right]
\end{array}
$$

where $\vec{f}_{n-1}$ is the mean value of $f_{n-1}$ throughout the shell being approximated by

$$
\bar{f}_{n-1}=-3 \frac{n+1}{n-1} \frac{n+m}{2 n+1} 4 \pi \sigma \xi_{n}^{m}(c) T_{2}(c) c^{2}
$$

Going back to condition (17), we may now determine $A_{n-1}$. We obtain

$$
A_{n-1}=-c^{n+1 / 2} \Delta c \bar{f}_{n-1}\left[K_{n-1 / 2}(k c)+I_{n-1 / 2}(k c) K_{n-3 / 2}(k a) / I_{n-3 / 2}(k a)\right]
$$

Putting (20) into (18), $s_{n-1}^{m}(r)$ is finally solved. In the following, only $s_{n-1}^{m}$ at $r=a$ is written down as

$$
s_{n-1}^{m}(a)=-\left(\frac{c}{a}\right)^{n-1 / 2} c \Delta c \bar{f}_{n-1} \frac{1}{k a} \frac{I_{n-1 / 2}(k c)}{I_{n-3 / 2}(k a)}
$$

With $s_{n-1}^{m}(a)$ thus determined, (15) enables us to obtain $i_{n-1}^{m}$, by which we can estimate the $S_{n-1}^{m}$ magnetic field emerging through the core's surface.

As for the $S_{n+1}^{m}$ field, a theory similar to the previous one leads to

$$
s_{n+1}^{\prime \prime \prime}(a)=-\left(\frac{c}{a}\right)^{n+3 / 2} c \Delta c \bar{f}_{n+1} \frac{1}{k a} \frac{I_{n+3 / 2}(k c)}{I_{n+1 / 2}(k a)}
$$

where

$$
\bar{f}_{n+1}=-3 \frac{n}{n+2} \frac{n-m+1}{2 n+1} 4 \pi \sigma \xi_{n}^{m}(c) T_{2}(c)
$$




\section{Steady state}

Making $p \rightarrow 0$ or $k \rightarrow 0$, we have

$$
\begin{aligned}
& I_{n-1 / 2}(k c) \sim \frac{1}{\Gamma\left(n+\frac{1}{2}\right)}\left(\frac{k c}{2}\right)^{n-1 / 2} \\
& I_{n-3 / 2}(k a) \sim \frac{1}{I\left(n-\frac{1}{2}\right)}\left(\frac{k a}{2}\right)^{n-3 / 2}
\end{aligned}
$$

Putting these into (21), we obtain

$$
s_{n-1}^{m}(a)=-\left(\frac{c}{a}\right)^{2 n} a \Delta c \frac{\bar{f}_{n-1}}{2 n-1} \quad \text { for } t \rightarrow \infty
$$

In the same way, we get

$$
s_{n+1}^{m}(a)=-\left(\frac{c}{a}\right)^{2 n+4} a \Delta c \frac{\bar{f}_{n+1}}{2 n+3} \quad \text { for } \quad t \rightarrow \infty
$$

It is easily seen that solutions (24) and (25) can be directly obtained from differential equations for the steady state.

Now we are in a position to estimate what velocity produces what magnetic field. Let us rewrite (23) as

$$
\bar{f}_{n+1}=-\frac{3}{(n+1)(n+2)} \frac{n-m+1}{2 n+1} 4 \pi \sigma \epsilon_{n, m}^{-1} v_{r}^{\prime} H_{0}^{\prime} c^{-n-1}
$$

where $v_{r}{ }^{\prime}$ multiplied by $\widetilde{Y}_{n}^{m}$ is the $r$ component of the velocity at $r=c$ as given by

$$
v_{r}^{\prime}=\left[n(n+1) \xi_{n}^{\prime \prime \prime} r^{n-1}\right]_{r=c} \epsilon_{n, m}
$$

In numerical work, it is more convenient to make use of spherical functions defined by Schmidt instead of those functions of Neumann's definition which have been adopted in the theory in the last section because, being partly normalized, Schmidt's functions are always less than unity. $\epsilon_{n, m}$ in the above expression is introduced because of the partial normalization and is given by

$$
\epsilon_{n, m}=\left\{\frac{(n+m) !}{2(n-m) !}\right\}^{1 / 2} \quad \text { for } \quad m \neq 0
$$

Meanwhile, we take

$$
H_{0}^{\prime}=\left[T_{2} r^{2}\right]_{r=c}
$$

so that, assuming that the maximum value (at $\theta=45^{\circ}$ ) of the stationary toroidal field as 300 gauss, $H_{0}^{\prime}$ is estimated as 200 gauss.

On the other hand, the coefficient for the $S_{n+1}^{m}$ field (Schmidt's function is again used) is given by

$$
i_{n+1}^{m}=\frac{3}{n+2} \frac{n-m+1}{(2 n+1)(2 n+3)} 4 \pi \sigma v_{r}^{\prime} H_{0}^{\prime} \Delta c\left(\frac{c}{a}\right)^{n+3} \frac{\epsilon_{n+1}, m}{\epsilon_{n \cdot m}}
$$


Suppose that there is a convectional motion of which the velocity is described by $n=5$ and $m=5$. (30) enables us to estimate $i_{6}^{5}$. Let us take

$$
\begin{aligned}
\sigma & =4 \times 10^{-6} \text { e.m.u. } \\
\Delta c & =0.1 a \\
c & =0.8 a \\
a & =3470 \mathrm{~km}
\end{aligned}
$$

In that case we see

$$
i_{n+1}^{m}=5.8 \Gamma
$$

for

$$
v_{r}{ }^{\prime}=0.01 \mathrm{~cm} / \mathrm{sec}
$$

The Gaussian coefficient of the potential at the earth's surface is then given by $\left(\frac{a}{a_{0}}\right)^{8} i_{0}^{5}$ ( $a_{0}$ : the earth's radius) which amounts to $0.045 \Gamma$. Judging from the observed magnitude of non-dipole fields, it is therefore possible to suppose that the velocity of the convection motion would be the order of $0.01 \mathrm{~cm} / \mathrm{sec}$ which hás been also suggested by Bullard and Gellman ${ }^{(13)}$ in their theory of geomagnetic dynamo. The induction process considered may well be responsible for the non-dipole fields.

\section{Non-steady state}

Going back to (21) or (22), which may be regarded as operational equations, we can examine the time-dependent behaviour of the $S_{n-1}^{m}$ and $S_{n+1}^{m}$ fields when some sudden change in the velocity takes place. If $\bar{f}_{n-1}$ changes instantaneously at $t=0$ and remains constant afterwards, we obtain

$$
s_{n-1}^{m}(a, t)=-\left(\frac{c}{a}\right)^{n-1 / 2} c \Delta c \bar{f}_{n-1} \frac{1}{2 \pi i} \int_{L} \frac{1}{\sqrt{4 \pi \sigma p} a} \frac{I_{n-1 / 2}(\sqrt{4 \pi \sigma p} c)}{I_{n-2 / 3}(\sqrt{4 \pi \sigma p a})} \frac{e^{p t}}{p} d p \quad \text { for } \quad t>0
$$

where $L$ is Bromwich's path of integration on the complex $p$-plane.

It is seen that the integrand has a branch point at $p=0$ and simple poles at

$$
p=-\alpha_{s}^{2} /\left(4 \pi \sigma a^{2}\right) \quad s=1,2,3, \ldots \ldots
$$

while $\alpha_{s}^{\prime} s$ are the roots of $J_{n-3 / 2}(z)=0$, so that

$$
J_{n-3 / 2}\left(\boldsymbol{\alpha}_{s}\right)=0 \quad 0<\boldsymbol{\alpha}_{1}<\boldsymbol{\alpha}_{2}<\boldsymbol{\alpha}_{3}<\ldots \ldots
$$

We take a path of integration as shown in Fig. 3 in which Bromwhich's path of integration is shown by the straight line along the imaginary axis, so that all the singularities lie on the lefthand-side of the path. The branch point and poles are also shown by $O, P_{1}, P_{2}, P_{3}, \ldots \ldots$. If we further consider the integration along the closed circuit like the one in Fig. 3, we get

$$
\int_{L}=\int_{O}+\int_{P_{1}}+\int_{P_{2}}+\int_{P_{3}}+\ldots \ldots
$$

where the integrals on the righthand-side are the ones integrated counterclockwise along the small circles centred at $O, P_{1}, P_{2}, P_{3}, \ldots \ldots$. It is readily proved that the 


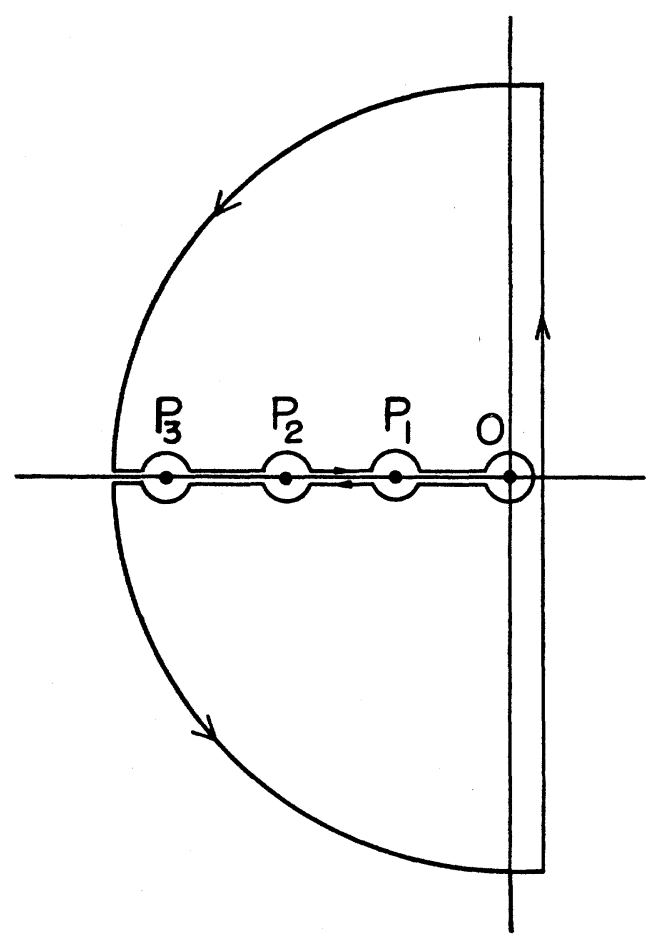

Fig. 3. The path of integration.

integrals along the large semi-circle vanishes when we make the radius infinite. Meanwhile, the integrals along the two straight lines (parallel to the real axis) taken on a Riemann-plane are also proved to cancel one another.

Putting (37), the integrals on its righthand-side being evaluated with the aid of the theory of residues, into (34), we obtain

$$
s_{n-1}^{m}(a, t)=F_{n-1}(t) s_{n-1}^{m}(a, \infty)
$$

where

$$
F_{n-1}(t)=1-4(2 n-1)\left(\frac{c}{a}\right)^{-n+1 / 2} \sum_{s=1}^{\infty} \frac{J_{n-1 / 2}\left(\frac{c}{a} \alpha_{s}\right)}{J_{n-1 / 2}\left(\alpha_{s}\right)-J_{n-5 / 2}\left(\alpha_{s}\right)} \frac{e^{-\frac{\alpha_{s}^{2} t}{4 \pi \sigma a^{2}}}}{\alpha_{s}^{2}}
$$

In a similar fashion, $s_{n+1}^{m}(a, t)$ is obtained as

$$
s_{n+1}^{m}(a, t)=F_{n+1}(t) s_{n+1}^{m}(a, \infty)
$$

where

$$
F_{n+1}(t)=1-4(2 n+3)\left(\frac{c}{a}\right)^{-n-3 / 2} \sum_{s=1}^{\infty} \frac{J_{n+3 / 2}\left(\frac{c}{a} \beta_{s}\right)}{J_{n+3 / 2}\left(\beta_{s}\right)-J_{n-1 / 2}\left(\beta_{s}\right)} \frac{e^{-\frac{\beta_{s}{ }^{2} t}{4 \pi \sigma a^{2}}}}{\beta_{s}^{2}}
$$

and

$$
J_{n+1 / 2}\left(\beta_{s}\right)=0 \quad 0<\beta_{1}<\beta_{2}<\beta_{3}<\ldots \ldots
$$

For small values of $t,(21)$ and (22) provide approximate equations like 


$$
\left.\begin{array}{l}
s_{n-1}^{m}(a, p)=-\left(\frac{c}{a}\right)^{n-1 / 2} c \Delta c \bar{f}_{n-1} \frac{e^{-k(a-c)}}{k a} \\
s_{n+1}^{m}(a, p)=-\left(\frac{c}{a}\right)^{n+3 / 2} c \Delta c \bar{f}_{n+1} \frac{e^{-k(a-c)}}{k a}
\end{array}\right\}
$$

so that we obtain

$$
\left.\begin{array}{l}
F_{n-1}(t)=(2 n-1)\left(\frac{c}{a}\right)^{-n+1 / 2}\left\{\frac{1}{a \pi} \sqrt{\frac{t}{\sigma}} e^{-\frac{(a-c)^{2} \pi \sigma}{t}}-\left(1-\frac{c}{a}\right)\left(1-\operatorname{erf} \frac{\sqrt{\pi \sigma}(a-c)}{\sqrt{t}}\right)\right\} \\
F_{n+1}(t)=(2 n+3)\left(\frac{c}{a}\right)^{-n-5 / 2}\left\{\frac{1}{a \pi} \sqrt{\frac{t}{\sigma}} e^{-\frac{(a-c)^{2} \pi \sigma}{t}}-\left(1-\frac{c}{a}\right)\left(1-\operatorname{erf} \frac{\sqrt{\pi \sigma}(a-c)}{\sqrt{t}}\right)\right\}
\end{array}\right\}
$$

which are sometimes convenient for numerical work.

Taking $n=5$, for example, the growth of the $S_{6}^{5}$ field is calculated. $F_{6}(t)$ as obtained from (41) and (44) is illustrated in Fig. 4. We see that the growth of the field is so

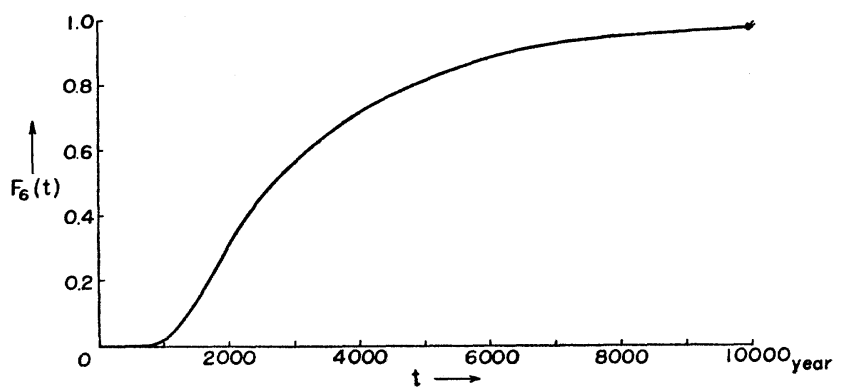

Fig. 4. Growth of the $S_{6}{ }^{5}$ field.

slow in the beginning that no appreciable field is observed until $10^{3}$ years past from the beginning. After that, the rate of increase gets larger, the 70 per cent of the steady field is attained after $4 \times 10^{3}$ years. After $10^{4}$ years, the steady state is nearly reached.

\section{Discussion and conclusion}

A simple estimate of the time required for transportation of a toroidal field by a motion of fluid, the conductivity of which is so high that the magnetic lines of force are frozen in it, indicates that the time is given by $d / v^{(12)}$ where $d$ is the distance and $v$ is the velocity. In order to bring up the toroidal field from a depth $0.8 a$ to the core's surface, it takes only 220 years for a velocity $0.01 \mathrm{~cm} / \mathrm{sec}$. From what we have been dealing with in the last seçtion, however, we see that it takes about 1400 years for the $S_{b}^{5}$ field to reach 10 per cent of its steady value. It seems misleading to define the time for the growth of poloidal fields simply by $d / v$ in this case. If transportation is made only by means of diffusion, it would take a time of order $4 \pi \sigma d^{2(12)}$ to diffuse from a depth $d$ through material of conductivity $\sigma$. Taking $\sigma=3 \times 10^{-6}$ e.m.u. as before, the time is about 7700 years. The induction process, therefore, seems to give a time for transportation shorter than the one in the case of simple diffusion 
process.

The curve in Fig. 4 indicates that the highest rate of increase for the radial component of the $S_{6}^{5}$ field is of the order of $10 \mathrm{r} / \mathrm{yr}$ at the earth's surface provided a $S_{5}^{5}$ motion having a velocity of the order of $0.01 \mathrm{~cm} / \mathrm{sec}$ is assumed in the core. If we assume $0.1 \mathrm{~cm} / \mathrm{sec}$ for the radial velocity, secular change of the order of $100 \mathrm{r} / \mathrm{yr}$ can be explained. According to the theory of geomagnetic dynamo(13), maximum radial and azimuthal velocities have been estimated as $0.014 \mathrm{~cm} / \mathrm{sec}$ and $0.04 \mathrm{~cm} / \mathrm{sec}$ respectively. For a more localized motion, it would not be impossible to suppose a velocity of the order of $0.1 \mathrm{~cm} / \mathrm{sec}$, so that the induction model considered here may account for the extremely intense foci of secular variation amounting to $200 \mathrm{r} / \mathrm{yr}$ at maximum in the Antarctic region.

The theory of induction has been in the above developed by specifying the fluid motion. Physically speaking, however, the driving force rather than fluid motion should have been specified because the pondermotive force produced by the growing magnetic field might become appreciable resulting in a considerable modification of the original motion. Such a dynamical consideration is a matter of great difficulty, no rigorous account based on exact magneto-hydrodynamics has been so far published. Even in this section, a very crude discussion of this point can be made in the following.

Among the various terms of the equation of motion for the core's fluid, it is known that the Coriolis force and the magneto-mechanical forces are the most important ${ }^{(1)}$, (13),(14). For a nearly steady flow, the magneto-mechanical force is then approximately balanced by the Coriolis force, the magnitude of which is given as $2 \omega \rho v$ ( $\omega$ : angular velocity of the earth's rotation, $\rho$ : density of the core's fluid). Taking $v=0.1 \sim 0.01 \mathrm{~cm} / \mathrm{sec}$, the Coriolis force and the magneto-mechanical force working on a unit volume of fluid are of order $10^{-4} \sim 10^{-5}$ dyn.

The most likely cause of the force that drives the convectional motion is believed to be the buoyancy force which is given as $\rho a g \delta T$, where $\alpha$ is the coefficient of cubical expansion, $g$ is the acceleration of gravity and $\delta T$ is the temperature departure from the surroundings. Putting $\alpha=4.5 \times 10^{-6}{ }^{\circ} \mathrm{C}^{-1}$ and $g=800 \mathrm{~cm} / \mathrm{sec}^{2}$, the buoyancy force becomes $0.04 \delta T$ as has been shown by Bullard and Gellman ${ }^{(13)}$. For the geomagnetic dynamo, it has been also shown by them that there is evidence to suppose that $\delta T$ is of the order of $10^{-3} \sim 10^{-4 \circ} \mathrm{C}$, so that the buoyancy force would be of order $10^{-5} \sim$ $10^{-6} \mathrm{dyn}$. It would not be also impossible to suppose that $\delta T$ is a little higher for localized eddies considered in this paper.

Although the estimate above is very crude, we may expect that the pondermotive force produced by the interaction between the induced electric currents and the stationary magnetic field could be of the same order of magnitude as that of the driving force. Since the pondermotive force is directed so as to counteract the fluid motion, the growth of the induced magnetic fields would become prohibited sooner or later because the motion is necessarily slowed down after some time when the pondermotive force is going to overcome the driving force. Whether such a process leads to an oscillation is not clear until a more detailed account of magneto-hydrodynamics becomes 
available. The fact that magnetic fields of many types are induced as we have seen in Section 2 makes the situation more difficult.

In the light of the above discussion, it seems doubtful that we have a monotonous approach to the steady state as shown in Fig. 4 in actual cases though the basic idea of induction model would still work in accounting for geomagnetic non-dipole fields and their secular variations.

\section{References}

(1) Elsasser, W.M., Phys. Rev., 69, 106, 1946; 70, 202, 1947; 72, 821, 1947.

(2) Bullard, E.C., M.N.R.A.S., Geophys. Suppl., 5, 248, 1948.

(3) Nagata, T., Oguti, T. and Kakinuma, S., Proc. Japan Acad., 34, 427, 1958.

(4) Nagata, T., and Syono, Y., J. Geomag. Geoele., 12, 84, 1960.

(5) Nagata, T., Antarctic Res., No. 11, 937, 1961.

(6) Bullard, E.C., Freedman, C., Gellman, H. and Nixon, J., Phil. Trans. R.S. London, A, 243, 67, 1950.

(7) Vestine, E.H., J. Geophys. Res., 58, 127. 1952.

(8) Nagata, T. and Oguti, T., J. Geomag. Geoelec., (Under Printing).

(9) Herzenberg, A. and Lowes, F.J., Phil. Trans. R.S. London, A, 249, 507, 1957.

(10) Coulomb, J., Rev. Fac. Sci. Univ. Istanbul, C., 19, 200, 1954. Ann. Geophys., 11, 80, 1955.

(11) Parker, E.N., Astrophys. J., 122, 293, 1955.

(12) Allan, D.W. and Bullard, E.C., Rev. Mod. Phys., 30, 1087, 1958.

(13) Bullard, E.C. and Gellman, H., Phil. Trans. R.S. London, A, 247, 213, 1954.

(14) Hide, R., Physics and Chemistry of the Earth 1, p. 94 (Pergamon Press, 1956). 